\title{
Serotype and antimicrobial profile distribution of invasive pneumococcal isolates in the pre-vaccine introduction era in Pretoria, South Africa, 2005 through 2009
}

\author{
Eduard Johan Silberbauer ${ }^{a,{ }^{\star}}$, Nazir Ismail ${ }^{a^{*}}$, Anne von Gottberg ${ }^{b}$, Anwar A. Hoosen $^{a}$ \\ ${ }^{a}$ Department of Medical Microbiology, University of Pretoria and National Health Laboratory Services, Tshwane Academic Division, \\ South Africa \\ ${ }^{b}$ Respiratory and Meningeal Pathogens Research Unit, National Institute for Communicable Diseases, Medical Research Council, \\ and University of the Witwatersrand, Johannesburg, South Africa
}

\begin{abstract}
A description of invasive Streptococcus pneumoniae isolates over a 5-year period from blood culture and cerebrospinal fluid culture follows, in Pretoria South Africa January 2005 through December 2009. Isolates were identified using standard microbiological techniques, serotyped, and a MIC determined for penicillin and cefotaxime. A total of 177 isolates were included in the analysis. Eighty percent of patients in the 18- to 45-year age group tested positive for HIV. In children b5 years of age, $66 \%(n=49)$ of serotypes were those present in the heptavalent pneumococcal conjugate vaccine (PCV-7). Fifty-nine percent $(n=29)$ were from PCV-7 serotypes in the b1-year-old age group. An additional peak of invasive disease was also seen in the 18- to 45-year age group. Only 1 of 177 isolates had resistance to penicillin $(\mathrm{MIC} \geq 2 \mu \mathrm{g} / \mathrm{mL}$ ); none was resistant to cefotaxime. The introduction of the PCV-7 vaccine in South Africa will decrease invasive pneumococcal disease caused by vaccine serotypes.
\end{abstract}

Keywords: South Africa; Streptococcus pneumoniae; Pneumococcus; Vaccine; Pneumococcus conjugate vaccine

Invasive pneumococcal disease has a classic biphasic distribution with toddlers and the elderly being mainly affected (Karstaedt et al., 2001). However, with the HIV pandemic this distribution pattern has been disturbed with a third peak in young- to middle-aged adults especially in countries with a high HIV burden such as South Africa (Jones et al., 1998).

Serotypes associated with invasive disease and higher MICs include the following: 1, 2, 3, 4, 6A, 6B, 9V, 14, 18C, 19F, and 23F (Karstaedt et al., 2000; Hausdorff et al., 2005). In late 2008, the PCV-7 conjugate vaccine was introduced into the South African Extended Programme for Immunisation (EPI) at a few pilot sites, with subsequent wider implementation to all primary health care clinics during 2009 (Madhi, 2008). Two primary doses are administered at 6 and 14 weeks with a booster dose administered at 9 months.

This study was carried out to describe the prevaccination serotypes in Pretoria for later postvaccination comparison. Serotypes and antimicrobial profiles of invasive pneumococcal isolates in the Pretoria region over a 5-year pre-vaccine introduction period were analysed.

Pneumococcal isolates from blood culture and cerebrospinal fluid (CSF) specimens submitted to the Microbiology Laboratory at the Tshwane Academic Division (National Health Laboratory Service) in Pretoria for the 5-year study period extending from January 2005 to December 2009 were analysed. Only single unique isolates per patient were used in the analysis.

* Corresponding authors. Eduard Johan Silberbauer is to be contacted at Tel.: +27123192338, Nazir Ismail, Tel.: +27123192252.

E-mail addresses: ejsilberbauer@gmail.com (E.J. Silberbauer), nazir.ismail@up.ac.za (N. Ismail). 
The laboratory serves 2 tertiary academic teaching hospitals (Steve Biko Academic Hospital, Kalafong Academic Hospital) and 3 district level hospitals (Tshwane District Hospital, Pretoria West Hospital, and Mamelodi Hospital).

Isolates were identified according to standard laboratory methods. MIC values for penicillin and cefotaxime were determined using the Etest ${ }^{\circledR}$ AB BIODISK (Solna, Sweden). MIC values were interpreted according to the Performance Standards for Antimicrobial Susceptibility Testing of the Clinical and Laboratory Standards Institute. Isolates were sent to the Respiratory and Meningeal Pathogens Unit at the National Institute for Communicable Diseases for serotyping. Isolates were serotyped by the Quellung method using specific antisera (Statens Serum Institut, Copenhagen, Denmark).

Isolates without sufficient data (patient age not recorded, MIC for penicillin or cefotaxime or serotyping not performed) were excluded from analysis.

Over the 5-year study period, there were a total of 192 single-patient isolates, 15 of which were excluded. Of the remaining 177 isolates, $49(28 \%)$ isolates were from children aged b5 years and 115 $(65 \%)$ from those aged N18 years (Table 1).

Of the 177 patients, HIV results were available in 79 patients. Of those tested, $16(70 \%)$ of 23 were positive for HIV in the b5-year age group. In the 18- to 45-year age group, 33 (81\%) of 41 tested positive for HIV.

Among the 49 isolates from the b5-year age group, 30 (61\%) were from the b1-year age group. Of these, 2 (4\%) were from b1 month, $19(38 \%)$ from 1-6 months, and $9(18 \%)$ from $6-12$ months.

The current PCV-7 vaccine serotypes (4, 6B, 9V, 14, 18C, 19F, 23F) accounted for $42 \%$ (75 of 177) of all isolates (Table 1). PCV-7 vaccine serotypes in the b5-year age group accounted for $67 \%$ (33 of 49 ) and specifically in the b1-year vaccine age group, 63\% (19 of 30) (data not shown). In the 18- to 45-year-old group, the PCV-7 vaccine serotypes accounted for $28 \%(n=21)$ of the whole group. A breakdown of the non-PCV-7 serotypes according to age groups is included in Table 1.

The MIC values for penicillin and cefotaxime are shown in Fig. 1. The majority, 170 (96\%), had a MIC value $\leq 0.5 \mu \mathrm{g} / \mathrm{mL}$ for penicillin. The remaining 7 isolates had a MIC of $1-2 \mu \mathrm{g} / \mathrm{mL}$ and included the following serotypes: $6 \mathrm{~A}(n=1), 14(n=3), 19 F(n=2)$, and 23F $(n=1)$. Two of these were blood culture isolates with a MIC of 1.5 and $2 \mu \mathrm{g} / \mathrm{mL}$. No isolate was resistant to cefotaxime, and $171(96 \%)$ had a MIC value of $\leq 0.5 \mu \mathrm{g} / \mathrm{mL}$. Six isolates (3\%) had a MIC of $1 \mu \mathrm{g} / \mathrm{mL}$ (serotypes: 3, 6B, 14, 19F, 23F).

The $\mathrm{MIC}_{50}$ and $\mathrm{MIC}_{90}$ for the antibiotics are shown in Table 2. The $\mathrm{MIC}_{50}$ values were higher for the PCV-7 serotypes.

In this study, PCV-7 serotypes were isolated in $66 \%$ of invasive pneumococcal disease (IPD) in the b5year age group. This is similar to what has been reported from developed countries such as Spain, as well as from developing countries such as The Gambia and South Africa (Perez-Trallero et al., 2009; Cutts et al., 2005; Zar et al., 2008). The introduction of the PCV-7 vaccine will likely lead to a significant reduction of IPD especially in our region with its high burden of HIV. The current South African EPI recommends that the PCV-7 vaccine be administered at 6 and 14 weeks with a booster dose at 9 months. A similar 2-dose primary series with one booster dose was shown to be immunogenic by Goldblatt et al. (2010).

A study in 2000 from South Africa reported $18.1 \%$ of invasive isolates from all ages and $28.9 \%$ from children b2 years of age in all regions of South Africa as resistant (MIC $\geq 2 \mu \mathrm{g} / \mathrm{mL}$ ) to penicillin (Huebner et al ., 2000). However, in this Pretoria study, only 1 isolate out of 177 was resistant $(\geq 2 \mu \mathrm{g} / \mathrm{mL})$ and all isolates were susceptible to cefotaxime. Thus, IPD in our area may still be adequately treated with penicillin.

We detected a high percentage (42\%) of IPD in the 18 to 45 year olds. This peak corresponds to the HIVIAIDS epidemiologic distribution in South Africa with the peak of HIV seropositivity at $31 \%$ in this age group (Department of Health, 2010). As expected, the HIV prevalence is increased in this population (81\%) to more than the antenatal surveillance data would suggest. The same was found in the b5-year age group with a HIV seropositivity of $70 \%$. The vaccination of the children with the PCV-7 vaccine may indirectly reduce the burden of disease among this vulnerable population. This has been noted in previous studies showing decreased colonization of children with serotypes causing IPD and thus decreased transmission to the adult age groups (CDC, 2005).

The serotypes included in the PCV-7 vaccine cover two thirds of the serotypes found in the b5-year age group, and the planned introduction of PCV-13 will be a welcome improvement. Furthermore, the low prevalence of fully penicillin-resistant isolates in the Pretoria region compared to national 


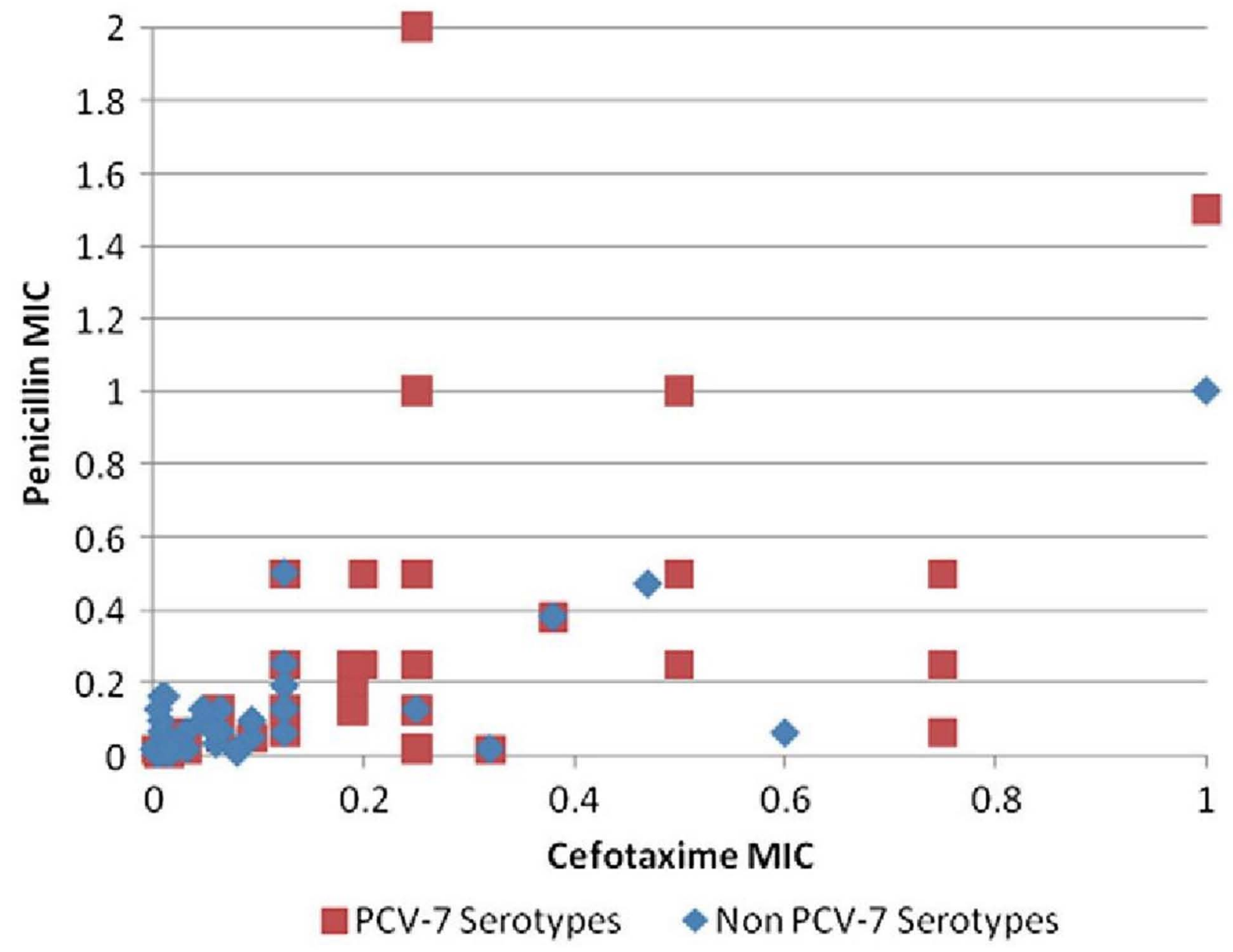

Fig. 1. Penicillin and cefotaxime MIC values according to vaccine serotypes $(n=177)$. 
Table 1

Serotypes according to age categories $(n=177)$

\begin{tabular}{lllllc}
\hline & $<5$ years & $6-17$ years & $18-45$ years & $\geq 45$ years & Total \\
\hline Vaccine serotypes & & & & \\
4 & 1 & 3 & 1 & 4 & $9(5 \%)$ \\
$6 \mathrm{~B}$ & 10 & 2 & 4 & 1 & $17(10 \%)$ \\
$9 \mathrm{~V}$ & 2 & 1 & 4 & 0 & $7(4 \%)$ \\
14 & 12 & 2 & 6 & 3 & $23(13 \%)$ \\
$18 \mathrm{C}$ & 1 & 0 & 0 & 0 & $1(0.6 \%)$ \\
$19 \mathrm{~F}$ & 2 & 2 & 2 & 2 & $8(4 \%)$ \\
$23 \mathrm{~F}$ & 5 & 0 & 4 & 1 & $10(6 \%)$
\end{tabular}

Nonvaccine serotypes

\begin{tabular}{llllll}
1 & 1 & 1 & 11 & 6 & $19(11 \%)$ \\
$6 \mathrm{~A}$ & 5 & 0 & 9 & 3 & $17(10 \%)$ \\
$9 \mathrm{~N}$ & 0 & 1 & 2 & 1 & $4(2 \%)$ \\
$12 \mathrm{~F}$ & 1 & 0 & 4 & 3 & $8(5 \%)$ \\
$19 \mathrm{~A}$ & 3 & 1 & 9 & 2 & $15(8 \%)$ \\
Other & $6^{*}$ & 0 & $19^{* *}$ & $14^{\#}$ & $39(22 \%)$ \\
Total & $49(28 \%)$ & $13(7 \%)$ & $75(42 \%)$ & $40(22 \%)$ & 177 \\
\hline
\end{tabular}

* $5(1) ; 8(1) ; 15 \mathrm{~A}(1) ; 29(1) ; 33 \mathrm{D}(2)$.

** 1 (11); 3 (3); 5 (1); 6C (1); 7F (1); 8 (2); 11A (1); 15F (2); $16(1)$; 17F (2); $20(1) ; 28(1) ; 29(1) ; 34$ (2).

\# $1(6) ; 3(5) ; 8(2) ; 10 \mathrm{~A}(2) ; 11 \mathrm{~F}(1) ; 13(1) ; 18 \mathrm{~A}(1) ; 22(1) ; 35 \mathrm{~B}(1)$.

Table 2

$\mathrm{MIC}_{50}$ and $\mathrm{MIC}_{90}$ for penicillin and cefotaxime $(n=177)$

\begin{tabular}{lllllll}
\hline Antibiotic & \multicolumn{2}{l}{$\mathrm{MIC}_{50}(\mu \mathrm{g} / \mathrm{mL})$} & & \multicolumn{2}{l}{$\mathrm{MIC}_{90}(\mu \mathrm{g} / \mathrm{mL})$} & $\begin{array}{l}\text { Range } \\
(\mu \mathrm{g} / \mathrm{mL})\end{array}$ \\
\cline { 2 - 3 } & $\begin{array}{l}\text { All } \\
\text { serotypes }\end{array}$ & $\begin{array}{l}\text { PCV } \\
\text { serotypes }\end{array}$ & & $\begin{array}{l}\text { All } \\
\text { serotypes }\end{array}$ & $\begin{array}{l}\mathrm{PCV} \\
\text { serotypes }\end{array}$ & \\
\hline Penicillin & 0.064 & 0.125 & & 0.500 & 0.500 & $0.004-2.000$ \\
Cefotaxime & 0.060 & 0.125 & & 0.380 & 0.500 & $0.002-1.000$ \\
\hline
\end{tabular}


surveillance data is also heartening (Huebner et al., 2000). Ongoing serotype and antimicrobial resistance monitoring following the introduction of the conjugate pneumococcal vaccine needs to continue as serotype replacement and emergence of resistant clones have been reported elsewhere (Messina et al., 2007).

\section{Acknowledgments}

The authors would like to thank the Tshwane Academic Medical Microbiology Laboratory (NHLS) for their work in providing the data for this publication and also the GERMS-SA (NHLS) group for providing the serotype data for this publication.

\section{References}

Centers for Disease Control and Prevention (CDC) (2005) Direct and indirect effects of routine vaccination of children with 7-valent pneumococcal conjugate vaccine on incidence of invasive pneumococcal disease-United States, 1998-2003. MMWR Morb Mortal Wkly Rep 54:893-897.

Cutts FT, Zaman SM, Enwere G, Jaffar S, Levine OS, Okoko JB, Oluwalana C, Vaughan A, Obaro SK, Leach A, McAdam KP, Biney E, Saaka M, Onwuchekwa U, Yallop F, Pierce NF, Greenwood BM, Adegbola RA, Gambian Pneumococcal Vaccine Trial Group (2005) Efficacy of nine-valent pneumococcal conjugate vaccine against pneumonia and invasive pneumococcal disease in The Gambia: randomised, double-blind, placebo-controlled trial. Lancet 365:1139-1146.

Department of health (2010) National antenatal sentinel HIV and syphilis prevalence in South Africa, 2009. Available at http://www.info.gov.za/ view/DownloadFileAction?id=109007. Last accessed 30 August 2011.

Goldblatt D, Southern J, Ashton L, Andrews N, Woodgate S, Burbidge P, Waight P, Miller E (2010) Immunogenicity of a reduced schedule of pneumococcal conjugate vaccine in healthy infants and correlates of protection for serotype 6B in the United Kingdom. Pediatr Infect Dis J 29:401-405.

Hausdorff WP, Feikin DR, Klugman KP (2005) Epidemiological differences among pneumococcal serotypes. Lancet Infect Dis 5:83-93.

Huebner RE, Wasas AD, Klugman KP (2000) Trends in antimicrobial resistance and serotypes distributions of blood and cerebrospinal fluid Isolates of Streptococcus pneumoniae in South Africa. Int $J$ Infect Dis 4:214-218.

Jones N, Huebner R, Khoosal M, Crewe-Brown H, Klugman K (1998) The impact of HIV on Streptococcus pneumoniae bacteraemia in a South African population. AIDS 12:2177-2184.

Karstaedt AS, Khoosal M, Crewe-Brown HH (2001) Pneumococcal bacteremia in adults in Soweto, South Africa, during the course of a decade. Clin Infect Dis 33:610-614.

Karstaedt AS, Khoosal M, Crewe-Brown HH (2000) Pneumococcal bacteremia during a decade in children in Soweto, South Africa. Pediatr Infect Dis J 19:454-457.

Madhi S (2008) Introduction of the pneumococcal conjugate vaccine into South African public immunization programme: dawn of a new era? South Afr $J$ Epidemiol Infect 23:05-09.

Messina AF, Katz-Gaynor K, Barton T, Ahmad N, Ghaffar F, Rasko D, McCracken GH Jr (2007) Impact of the pneumococcal conjugate vaccine on serotype distribution and antimicrobial resistance of invasive Streptococcus pneumoniae isolates in Dallas, TX, children from 1999 through 2005. Pediatr Infect Dis J 26:461-467.

Perez-Trallero E, Marimon JM, Ercibengoa M, Vicente D, Perez-Yarza EG (2009) Invasive Streptococcus pneumoniae infections in children and older adults in the north of Spain before and after the introduction of the heptavalent pneumococcal conjugate vaccine. Eur J Clin Microbiol Infect Dis 28:731-738.

Zar HJ, Madhi SA (2008) Pneumococcal conjugate vaccine-a health priority. S Afr Med J 98:463-467. 